Apidologie, 1972, 3 (2), 105-124.

\title{
OBSERVATIONS SUR LA POLLINISATION DE LA LUZERNE PAR LES ABEILES (APIS MELLIFICA L.) EN ZONE ARIDE IRRIGUEE AU MAROC
}

\author{
Beobachtungen über die Bestäubung der Luzerne durch die Biene \\ (Apis mellifica L.) \\ in den bewässerten Dürregebieten Marokkos
}

\section{J.-N. TASEI}

Laboratoire d'Éthologie et d'Écologie des Insectes, I.N.R.A., 86-Lusignan

\section{SUMMARY}

\author{
LUCERNe POLlination BY HONEY-BEES (Apis mellifica L.) IN \\ IRRIGATED ARID LANDS OF MOROCCO
}

The foraging of honey-bees (Apis mellifica intermissa) was observed in October 1969 in an irrigated lucerne field sown for seed (Medicago sativa L.) in the arid district of Beni Mellal (Morocco).

$86 \%$ of the bees were pollen-gatherers. Their tripping rate reached 20 percent of the visited flowers. The foraging speed was 4 flowers/minute.

The behaviour of these bees, nesting in local hives, was compared with the behaviour of a colony removed from Rabat (a relatively temperate district). This colony gathered only the pollen of several cultivated plants and weeds (Zea maïs, Amaranthus, Casuarina, Picris, Datura...)

\section{RESUME}

L'auteur a observé en octobre 1969, dans la région aride de Beni Mellal (Maroc), le butinage des abeilles (Apis mellifica intermissa) sur une culture porte-graines irriguée de luzerne (Medicago sativa L.)

Les récolteuses de pollen de luzerne représentaient $86 \%$ de la population des butineuses. Le pourcentage de déclenchement était $20 \%$ du total des fleurs visitées. Les abeilles visitaient en moyenne 4 fleurs par minute.

Le comportement de ces abeilles issues d'un rucher local fixe a été comparé à celui des abeilles issues d'une ruche originaire de la région relativement tempérée de Rabat. Ces dernières ont recherché uniquement le pollen de plantes compétitrices cultivées ou spontanées (Zea maïs, Amaranthus, Casuarina, Picris, Datura...) 


\section{I. - INTRODUCTION}

Dans plusieurs pays, les chercheurs qui se sont penchés sur le problème de la pollinisation de la luzerne ont montré que l'efficacité pollinisatrice de l'abeille domestique est assez variable et en moyenne faible (ВонART, 1957 - Lecomte, 1959).

Le manque d'affinité des abeilles pour le pollen de luzerne est généralement expliqué par l'effet répulsif du déclenchement de la colonne sexuelle des fleurs et par l'absence de substances attractives qui seraient présentes dans les pollens des fleurs compétitrices.

Tous les auteurs, en accord avec Vansell et Todd (1946) et ReinhardT (1952) distinguent 3 catégories de butineuses :

a) les récolteuses de nectar vieilles et « expérimentées » qui prélèvent le nectar latéralement et déclenchent 0 à $5 \%$ des fleurs;

b) les récolteuses de nectar jeunes et c inexpérimentées » qui déclenchent $50 \%$ des fleurs en prélevant le nectar par le centre de la corolle;

c) les récolteuses de pollen déclenchant 30 à $80 \%$ des fleurs mais visitant 2 fois moins de fleurs que les 2 premières catégories.

Les butineuses de nectar expérimentées, peu efficaces, sont les plus nombreuses. Cependant, certaines conditions de culture aux U.S.A. et en Hongrie permettent aux butineuses de type $b$ et $c$ de jouer un rôle dans la production de semences de luzerne.

Les auteurs font état de facteurs influant sur l'efficacité et le nombre des abeilles domestiques : abondance de la flore compétitrice, qualité et quantité du nectar, déplacement de ruches d'une région à une autre etc...

Au cours de l'année 1969, nous avons recueilli quelques renseignements sur le comportement pollinisateur de l'abeille domestique (Apis mellifica intermissa) dans les luzernières marocaines, en évaluant l'influence de certains facteurs d'activité.

\section{II. - OBSERVATIONS PRÉliminaIRES}

Le comportement de l'abeille domestique a été observé dans deux localités pendant les mois de juin, juillet et août, sur des surfaces de luzerne assez réduites de l'ordre d'un hectare.

10 Dans la région littorale et tempérée de Rabat, sur le domaine expérimental d'El Koudia et tout au long de la floraison de la luzerne, nous n'avons 
jamais surpris un individu portant des pelotes de pollen de luzerne ou déclenchant une fleur. Les butineuses visitaient la luzerne seulement pour son nectar. Les pollens disponibles à cette époque étaient fournis par une floraison échelonnée et abondante de Composées, Papilionacées, Liliacées, Borraginacées et Myrtacées.

$2^{\circ}$ Dans la région aride de Settat à Boulaouane au cours de deux journées d'observation, à la fin du mois de juin, sur luzerne irriguée et non irriguée (variété Indienne) nous avons constaté que certains individus déclenchaient un pourcentage variable des fleurs visitées. D'autre part sur 784 abeilles dénombrées, 71 portaient des pelotes de pollen (41 chargements analysés étaient du pollen de luzerne pur exception faite de 5 constitués de pollen de luzerne mélangé avec du pollen d'Eucalyptus (type gomphocephalus) et du pollen d'Asparagus albus. Le maximum de population a butiné entre 9 heures et 10 heures 30. La flore environnante (Composées, Myrtacées, Heliotropium), était très clairsemée.

Afin de préciser ces indications, nous avons observé de manière plus détaillée l'activité pollinisatrice des abeilles domestiques.

\section{III. - MATERIEL ET METHOdES}

Ces observations détaillées ont été effectuées uniquement dans la ferme expérimentale de Deroua près de Beni Mellal sur le plateau du Tadla, lors d'une floraison tardive de la luzerne pendant le mois d'octobre. Les figures 1 et 1 bis permettent de comparer les caractéristiques climatiques de la région de Rabat à celles de la région de Beni Mellal. Il ressort de la comparaison que de mai à octobre, le climat de Beni Mellal est beaucoup plus chaud et sec que celui de Rabat. Il est considéré comme aride. La figure 2 représente les relevés climatiques relatifs à la période d'observation.

Les abeilles domestiques sont observées durant 5 journées du début à la fin de leur période d'activité sur luzerne : les 7, 14, 22, 29 octobre et le 5 novembre 1969. Le champ d'observation est une parcelle de 11 ha de variété Sud Africaine, qui a été irriguée juste après la dernière coupe. Les plantes sont plus denses que dans les cultures porte-graines ordinaires. La hauteur de la végétation est 50 à $70 \mathrm{~cm}$. L'irrigation a favorisé dans la culture le développement d'Amaranthus, Convolvulus, Picris, etc. La population locale d'abeilles niche dans 3 à 4 centaines de ruches en poterie (djebaa traditionnelles) disposées à 2 ou 300 mètres de la luzerne. Afin de récolter le pollen ramené par les ouvrières, nous avons transporté le 7 octobre en bordure du champ une ruche “ DADANT » en bon état, appartenant au rucher du domaine d'El Koudia (Rabat) et munie d'une trappe à pollen. On sait que la trappe à pollen a été utilisée pour étudier le comportement de butinage de colonies d'abeilles domestiques, par de nombreux auteurs : Hape et Vanseld, (1946) - LouveauX, (1958) - Steuckardt, (1962) - Louveaux et al, (1966), etc.

Une partie des observations porte sur les fleurs de luzerne. L'importance de la floraison est évaluée grâce au comptage des inflorescences emprisonnées dans un cercle de $50 \mathrm{~cm}$ de diamètre lancé au hasard 10 fois sur la végétation. La concentration du nectar en matières sèches est mesurée au réfractomètre après pipetage dans une ou plusieurs fleurs. Ces contrôles de concentration ont lieu à toutes les heures de la journée.

On note le pourcentage des fleurs dépourvues de nectar.

Pendant la période d'observation, un inventaire de la flore environnante est fait. 

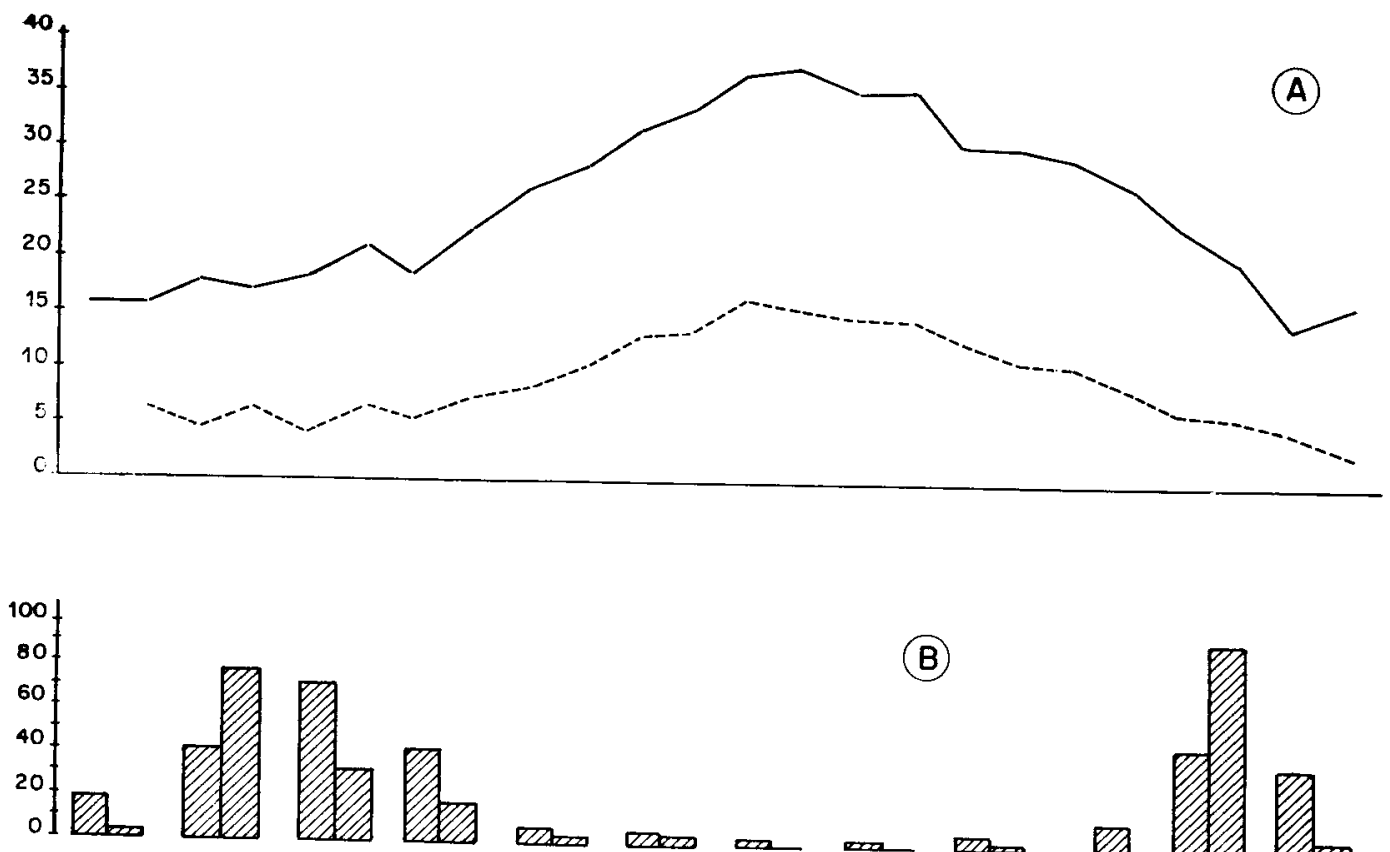

(B)
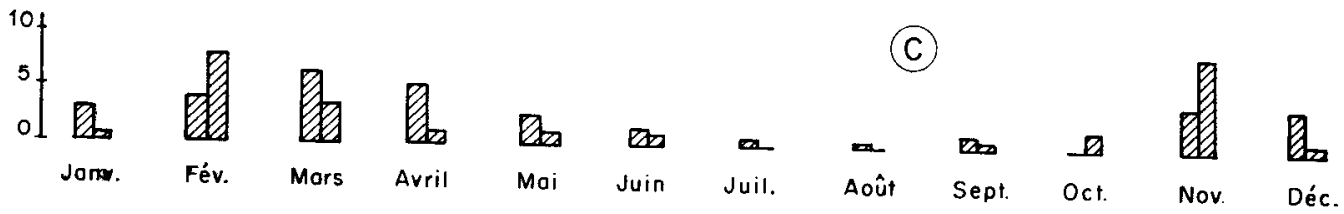

Fig. 1. - Relevês climatologiques 1968-69 à Déroua (Beni Mellal).
A. - Températures par quinzaine trait plein $=$ Moyenne des maxima en ${ }^{\circ} \mathrm{C}$.
trait pointillé $=$ Moyenne des minima en ${ }^{\circ} \mathrm{C}$
B. - Précipitations par quinzaine en $\mathbf{m m}$.
C. - Nombre de jours de pluie par quinzaine.

Aв8. 1. - Klimaangaben 1968-69 in Déroua (Beni Mellal) :
A. - Temperaturen (14 tägig); ausgezogene Linie $=$ durchschnittl. Höchsttemperatur in ${ }^{\circ} \mathrm{C}$, unterbrochene Linie $=$ durch-
B. - Niederschlag in $\mathrm{mm}, 14$ tägig
C. - Regentage (2 Wochen). 
(A)
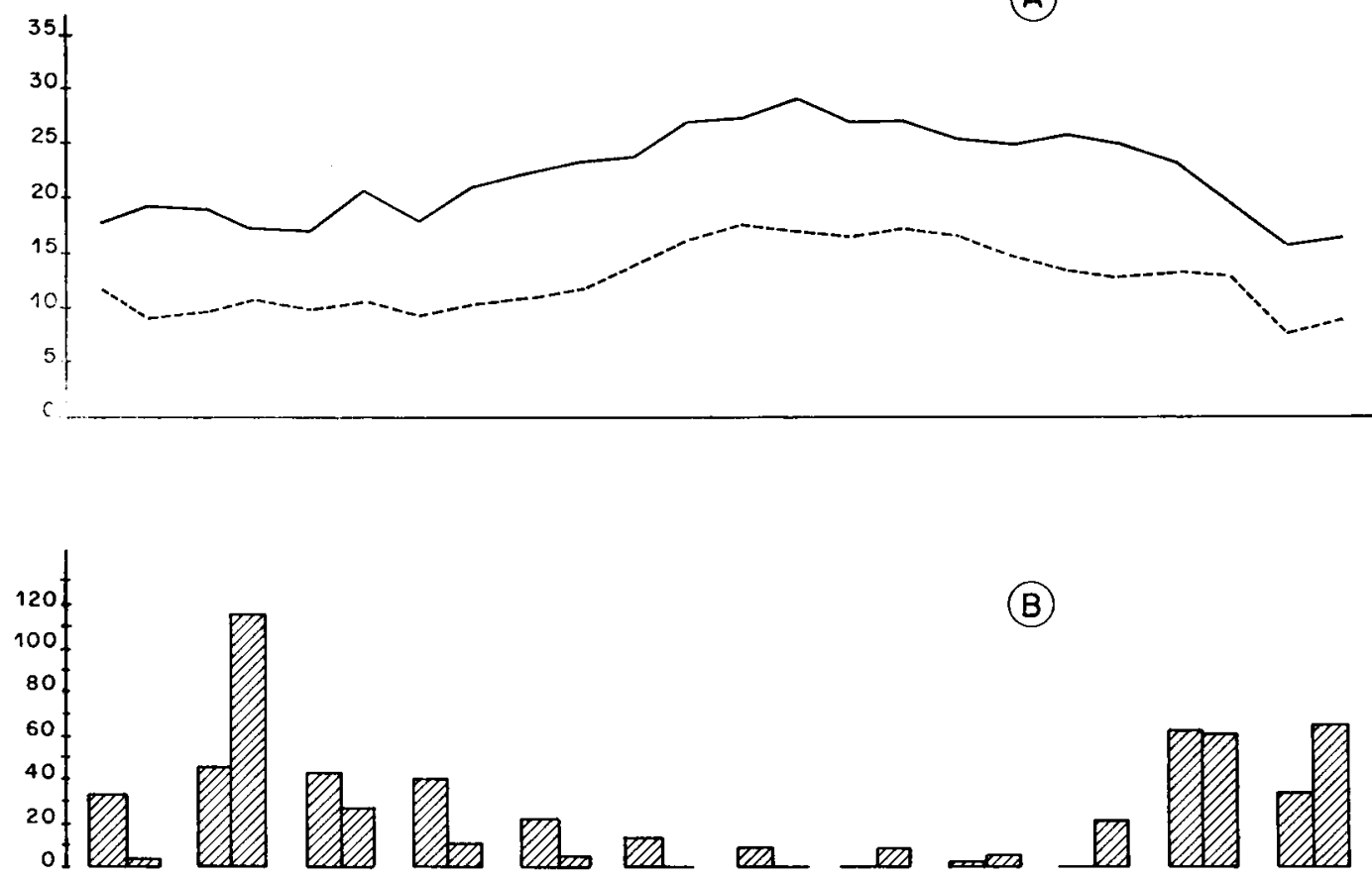

(B)
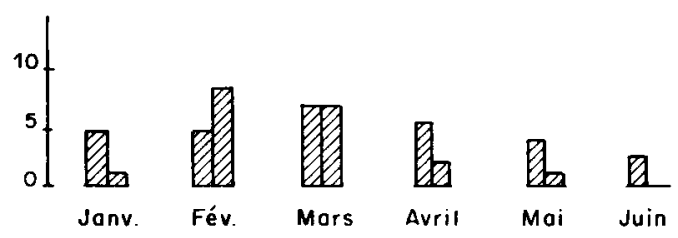

Juil.

$\rightarrow$

Sept.

(C)

Fig. 1 bis. - Relevés climatologiques 1968-69 du C.I.D.E.R.A. (Rabat).
A. - même chose que fig. 1 .
B. - même chose que fig. 1 .
C. - même chose que fig. 1 .

Aв8. 1. - (Fortsetzung) Klimaangaben 1968-69 d. C.I.D.E.R.A. (Rabat).

A, B, C wie oben. 


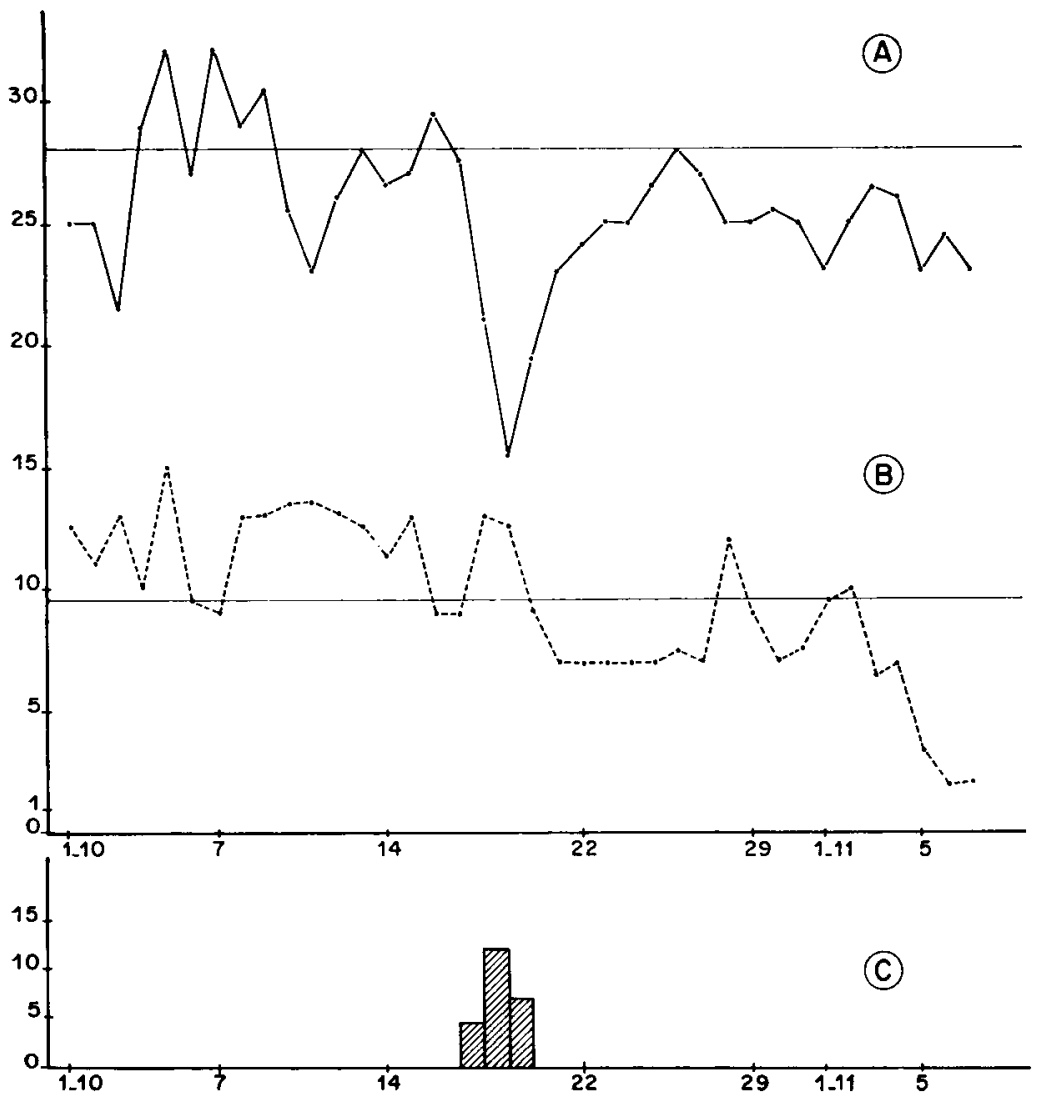

Fic. 2. - Relevés climatologiques octobre-novembre 1969 Déroua (Beni Mellal).
A. - Températures maximales par jour en ${ }^{\circ} \mathrm{C}$. Le trait horizontal $=$ moyenne mensuelle.
B. - Températures minimales par jour en ${ }^{\circ} \mathrm{C}$. Le trait horizontal $=$ moyenne mensuelle.
C. - Précipitations en mm.

Авв. 2. - Klimaangaben Oktober/November 1969 Déroua (Beni Mellal) :
A. - Höchsttemperatur pro Tag in ${ }^{\circ} \mathrm{C}$;
Horizontale $=$ wöchentlicher Durchschnitt;
B. - Tiefsttemperaturen je Tag in ${ }^{\circ} \mathrm{C}$; Horizontale wie oben;
C. - Niederschlag in mm.

Les renseignements acquis sur les abeilles portent sur les variations de l'activité de butinage. Chaque heure de la journée, la population d'abeilles est évaluée en comptant le nombre d'individus butinant sur la bordure fleurie de $100 \mathrm{~m}$ de longueur et de $1 \mathrm{~m}$ de largeur, suivant la méthode d'évaluation des populations de pollinisateurs recommandée et utilisée par LECoMTE, 1962; BENEDEK, 1970; TAseI (sous-presse). Les individus portant des chargements de pollen sont comptés à part. Quelques abeilles sont capturées au cours de la journée pour vérifier l'identité du pollen récolté (cf. : ANasiewiCz et WarakomsKa, 1969). 
D'autre part, le comportement individuel d'une cinquantaine d'abeilles portant des pelotes a été observé. Nous avons compté le nombre de fleurs visitées pendant 250 minutes, en distinguant les fleurs vierges et les fleurs déjà déclenchées. La vitesse et le pourcentage de déclenchement sont calculés à partir de ces comptages. Enfin, toutes les heures, le contenu de la trappe à pollen est prélevé, puis trié pelote après pelote. L'identification des espèces s'effectue grâce aux couleurs des pelotes et à des analyses microscopiques permettant de confirmer ou de rectifier le classement des pelotes. LouveauX (1958) estime que moyennant ces précautions les résultats sont précis.

Les préparations microscopiques de pollen s'effectuent suivant la technique de Mavrizio et Louveaux (1965) légèrement modifiée : après lavage dans un mélange d'éther et d'alcool, un échantillon du chargement complet porté par chaque insecte capturé est monté entre lame et lamelle dans une goutte de gélatine glycérinée, légèrement colorée à la fuchsine et liquéfiée à la chaleur. L'analyse de ces préparations s'effectue par comparaison avec des lames de référence faites à partir des étamines de plantes fraîches.

Pendant toute la durée des observations, la température et l'humidité de l'atmosphère font l'objet d'un enregistrement sous l'abri météorologique de la ferme à 2 mètres au-dessus du sol.

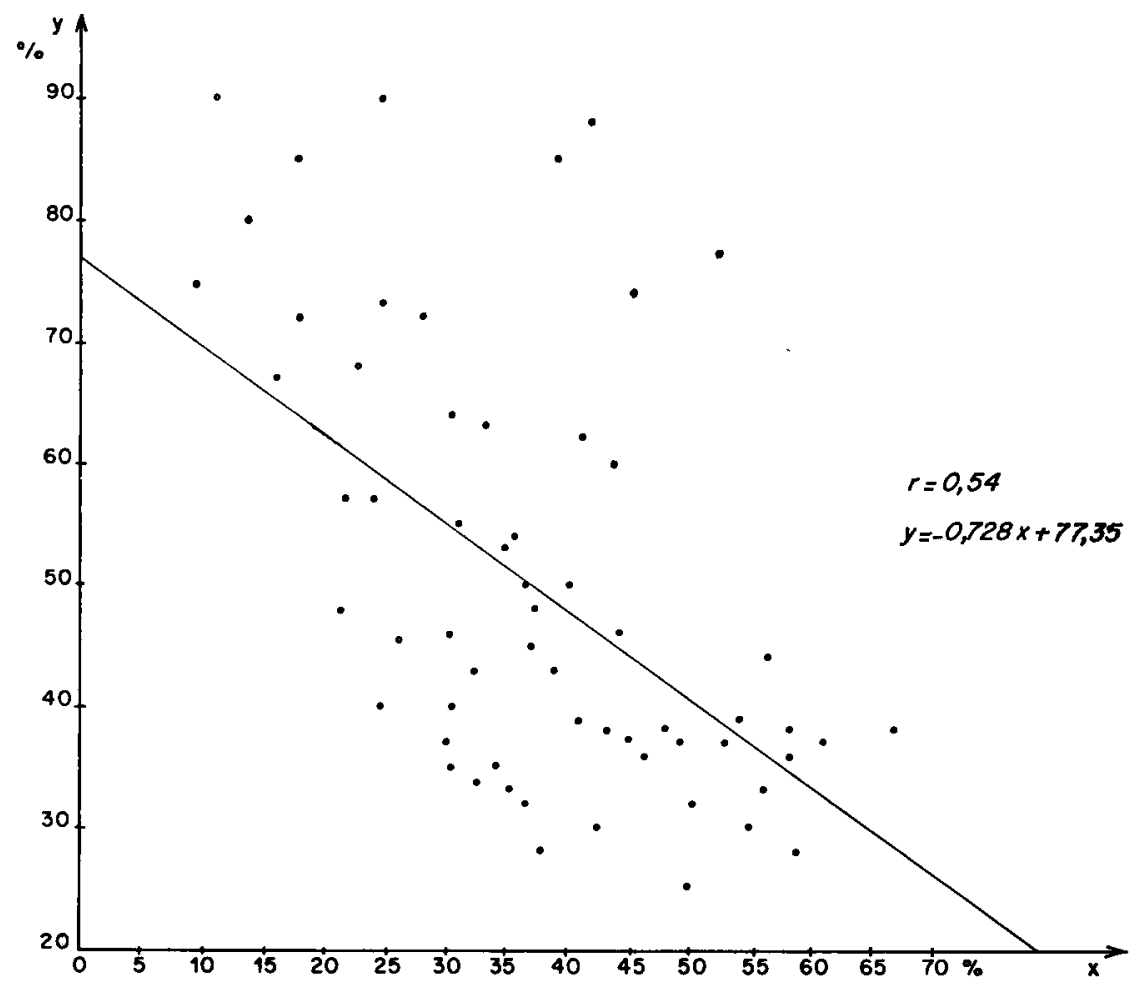

Fig. 3. - Humiditê de l'air (axe des y) et concentration en sucres du nectar de luzerne (axe des $x$ ) à Déroun (Beni Mellal) octobre-novembre 1969.

Aвв. 3. - Luftfeuchtigkeit (y*Achse) und Zuckerkonzentration des Luzernenektars ( $x$-Achse) in Déroun (Beni Mellal) Oktober / November 1969. 


\section{IV. - RESULTATS}

\section{A. - La floraison de la luzerne, la sécrétion de nectar}

La floraison tardive est très peu abondante (19 à 25 inflorescences au $\mathbf{m}^{2}$ ) en raison des températures nocturnes basses (fig. 2), de la forte densité des plantes (luzerne et adventices), et probablement de la longueur du jour.

La quantité de nectar sécrétée semble être affectée par l'abaissement des températures nocturnes et diurnes au cours du mois d'octobre. C'est ainsi que le 29 octobre 1969 nous avons pu prélever du nectar dans $18 \%$ des fleurs (212 fleurs) et le 5 novembre 1969 dans $8 \%$ seulement (721 fleurs).

Les moyennes de concentration du nectar au cours des 4 journées d'observation sont assez stables : $40 \%-36 \%-35 \%-43 \%-43 \%$. Les concentrations individuelles varient de $9 \%$ à $67 \%$. Il existe une corrélation positive faiblement significative $(r=+0,38)$ entre la concentration du nectar et la température. Entre cette concentration et le degré d'humidité de l'atmosphère, la corrélation négative est significative et bonne $(r=-0,54$ ) (fig. 3 ).

\section{B. - La floraison des autres plantes pollinifères}

Au cours du mois d'octobre, les fleurs disponibles pour les abeilles sont assez rares. Certaines poussent spontanément dans les parcelles irriguées ou le long des seguias : Solanum niger, Datura, Hirschfeldia incana, Convolvulus arvensis, Marrubium vulgare, Amaranthus albus, Amaranthus hybridus, Picris echioides, Erigeron canadense, Lactuca, Calendula, Pulicaria arabica. D'autres sont cultivées dans 2 parcelles proches de la luzerne : Gossypium, Hibiscus cannabinus. Enfin Eucalyptus, Schinus molle, Bougainvillea, Zea maìs, se rencontrent à la limite du domaine.

\section{C. - Le comportement des abcilles domestiques}

La population des butineuses a diminué progressivement du 7 octobre au 5 novembre.

Le 7-10 ont été dénombrées sur la zone de comptage : 200 abeilles

Le 14-10 ont été dénombrées sur la zone de comptage : 66 abeilles

Le 22-10 ont été dénombrées sur la zone de comptage : 28 abeilles

Le 29-10 ont été dénombrées sur la zone de comptage : 21 abeilles

Le 5-11 ont été dénombrées sur la zone de comptage : 17 abeilles 
La figure 4 met en évidence la période d'activité de butinage sur la luzerne : entre 9 heures 30 et 14 heures 30 avec un maximum à 10 heures 30 .

Cette population d'abeilles est constituée de $91 \%$ (332 abeilles) d'individus porteurs de pelotes (butineuses de pollen). Cette proportion est restée assez stable au cours des observations, ne variant que de $81 \%$ à 94\%. 96 chargements de pollen ont été prélevés au hasard et leur analyse a révélé que 91 étaient composés de pollen de luzerne pur, 4 de pollen de Composées pur et 1 de pollen d'Amaranthus pur. On peut estimer à $86 \%$ le pourcentage de butineuses récoltant du pollen de luzerne.

Le comportement individuel de butinage des récolteuses de pollen a été observé. Elles prélèvent le pollen de luzerne sur les fleurs déjà déclenchées aussi bien que sur les fleurs vierges qu'elles déclenchent. Elles aspirent du nectar sur les fleurs des 2 types avant de gratter le pollen avec leurs pattes. Sur les fleurs vierges, le nectar est récolté de façon frontale, le proboscis étant introduit au centre de la corolle, mais le plus souvent l'ouvrière fait glisser son proboscis latéralement entre l'étendard et les ailes sans risquer d'écarter la carène. Certaines ouvrières semblent avoir ( un plan » de butinage établi : par exemple, série de fleurs déjà déclenchées où sont prélevés nectar et pollen, puis série de fleurs vierges où est prélevé le nectar sans déclenchement, enfin série de fleurs vierges où est prélevé le pollen après déclenchement.

Lors du déclenchement, ou bien l'ouvrière aborde la fleur de façon frontale, face à l'étendard et écarte la carène en enfonçant son proboscis au fond de la corolle, ou bien elle aborde la fleur en position inversée, face à la carène, et écarte l'étendard qu'elle tient entre les pattes pendant qu'elle plonge ses pièces buccales dans la corolle, ou bien encore elle aborde la fleur perpendiculairement à son axe de symétrie et provoque le déclenchement grâce à la pression exercée par le proboscis.

Souvent, l'insecte éprouve quelques difficultés à retirer sa langue coincée entre l'étendard et la colonne sexuelle qu'il vient de déclencher. Pendant 250 minutes, 50 ouvrières ont visité 1045 fleurs. La vitesse de butinage est donc de 4,1 fleurs par minute. $62,7 \%$ des fleurs visitées étaient vierges, $37,3 \%$ étaient déjà pollinisées. Les ouvrières ont déclenché $31,4 \%$ des fleurs vierges, soit $19,8 \%$ du total des fleurs visitées. Ia vitesse de déclenchement est de 0,8 fleur par minute. Les variations individuelles de comportement du lot d'abeilles observées sont représentées par les histogrammes de fréquence de la figure 5 . 

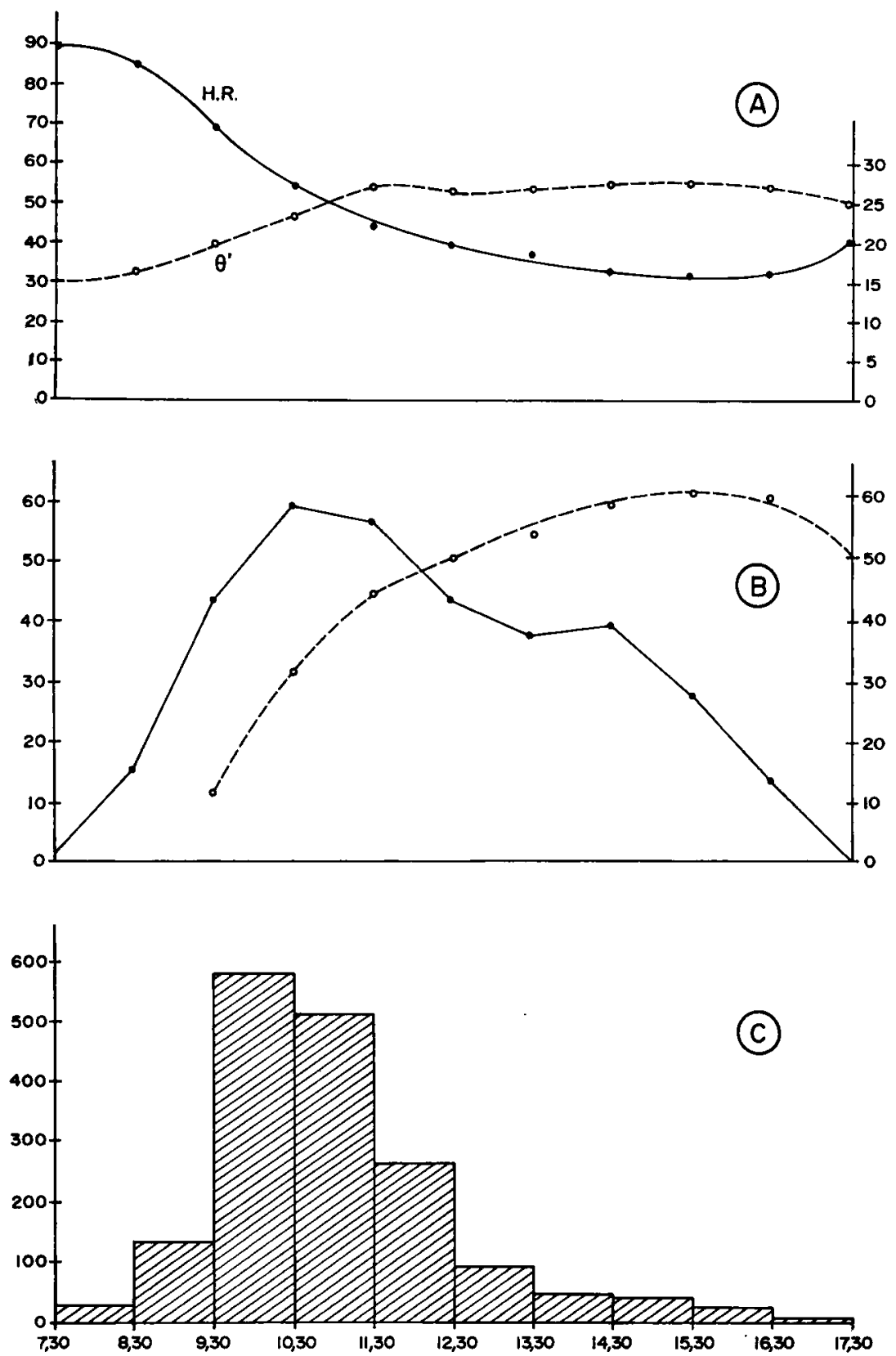

Fig. 4. - Évolution moyenne au cours de la journée (axe des $x=$ heures) :

A. - de la température et de l'humidité relative de l'air;

B. - de la population d'abeilles domestiques sur la luzerne (trait plein) et de la concentration du nectar de luzerne (trait pointillé) ;

C. - de la récolte de pollen de plantes compétitrices, à Déroua (Beni Mellal) octobre 1969.

ABB. 4. - Durchschnittsentwicklung im Verlauf eines Tages $(x$-Achse $=$ Stunden) :

A. - der Temperatur und Luftfeuchtigkeit;

B. - des Bienenbefluges auf der Luzerne (ausgezogene Linie) und der Nektarkonzentration der Luzerne (unterbrochene Linie);

C. - der Pollenernte konkurrierender Pollenspender in Déroua (Beni Mellal) Oktober 1969. 
D. - La récolte de pollen des abeilles provenant de la région de Rabat

Le 7-10-69, jour d'installation de la ruche, aucun transport de pollen n'est observé. Puis sont dénombrées :

$$
\begin{aligned}
& \text { - le 14-10: } 156 \text { pelotes } \\
& \text { - le 22-10:606 pelotes } \\
& \text { - le 29-10:530 pelotes } \\
& \text { - le 5-11 : } 596 \text { pelotes }
\end{aligned}
$$

Aucune pelote de pollen de luzerne n'est recueillie dans la trappe, bien que la taille des pelotes observées sur les abeilles locales soit normale. Les pollens rencontrés appartiennent aux plantes suivantes : Convolvulus Gossypium - Picris - Lactuca - Zea - Eucalyptus - Amaranthus Solanum - Erigeron - Datura - Hirschfeldia - Hibiscus - Bougainvillea Casuarina -2 plantes indéterminées.

La figure 6 met en évidence les différentes quantités de pelotes récoltées suivant l'espèce de plante. Les 5 pollens principaux sont : Zea maïs, Casuarina, Amaranthus, Picris et Datura; Zea maïs étant de loin le plus abondant. Le tableau 1 indique l'évolution des proportions de la récolte de ces 5 pollens, entre le 14-10 et le 5-11. Notons que la récolte sur maïs croît régulièrement
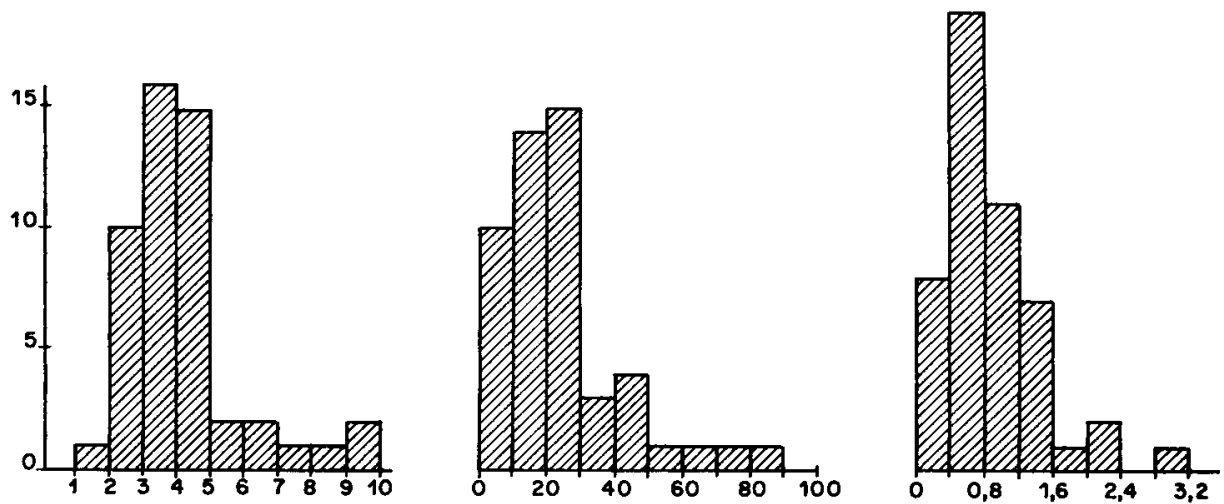

Fig. 5. - Comportement de butinage de l'abeille domestique locale sur luzerne à Déroua (Beni Mellal); octobre 1969.

A. - Rapidité de butinage : axe des $x=$ Nombre de fleurs par minute. axe des $y=$ Nombre d'abeilles.

B. - Pourcentage de fleurs déclenchées.

C. - Vitesse de déclenchement en nombre de fleurs par minute.

Aвв. 5. - Sammelverhalten der lokalen Honigbiene auf der Luzerne in Déroua (Beni Mellal) Oktober 1969

A. - Sammelgeschwindigkeit; $x$-Achse $=$ Zahl d. Blüten je Minute; y-Achse $=$ Zahl d. Bienen;

B. - Prozentsatz d. " aufgeklinkten " Blüten;

C. - Geschwindigkeit des « Aufklinkens » in Blütenzahlen je Minute. 


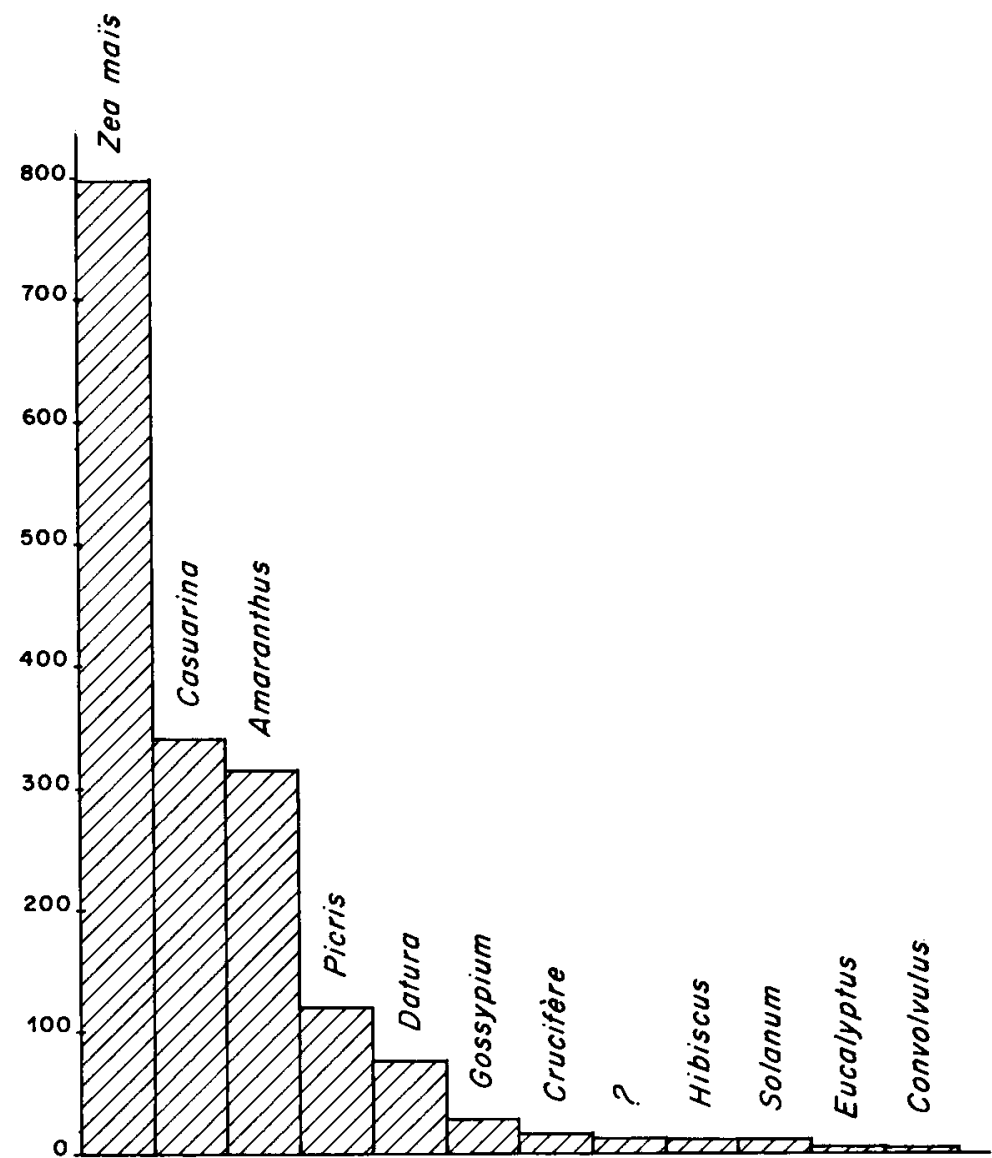

Fic. 6. - Récolte de pollen de plantes compétitrices par une colonie originaire de Rabat Déroua (Beni Mell. I): octobre 1969.

Axe des $y=$ nombre de pelotes.

Aв8. 6. - Pollenernte eines aus Rabat stammenden Bienenvolkes von anderen Pollenspendern; Oktober 1969 : y-Achse $=$ Zahl der Pollenhöschen.

jusqu'à représenter près des $3 / 4$ des pelotes. La récolte sur Amaranthus suit une évolution inverse. Quant au pollen de Casuarina d'ordinaire relativement peu récolté, il représente la moitié du contenu de la trappe le 22-10.

L'analyse du relevé horaire de la trappe apparaît sur la figure 4. En moyenne, la majeure partie des pollens est récoltée entre 9 heures 30 et 12 heures 30 avec un maximum d'activité entre 9 heures 30 et 10 heures 30 . Cependant, 


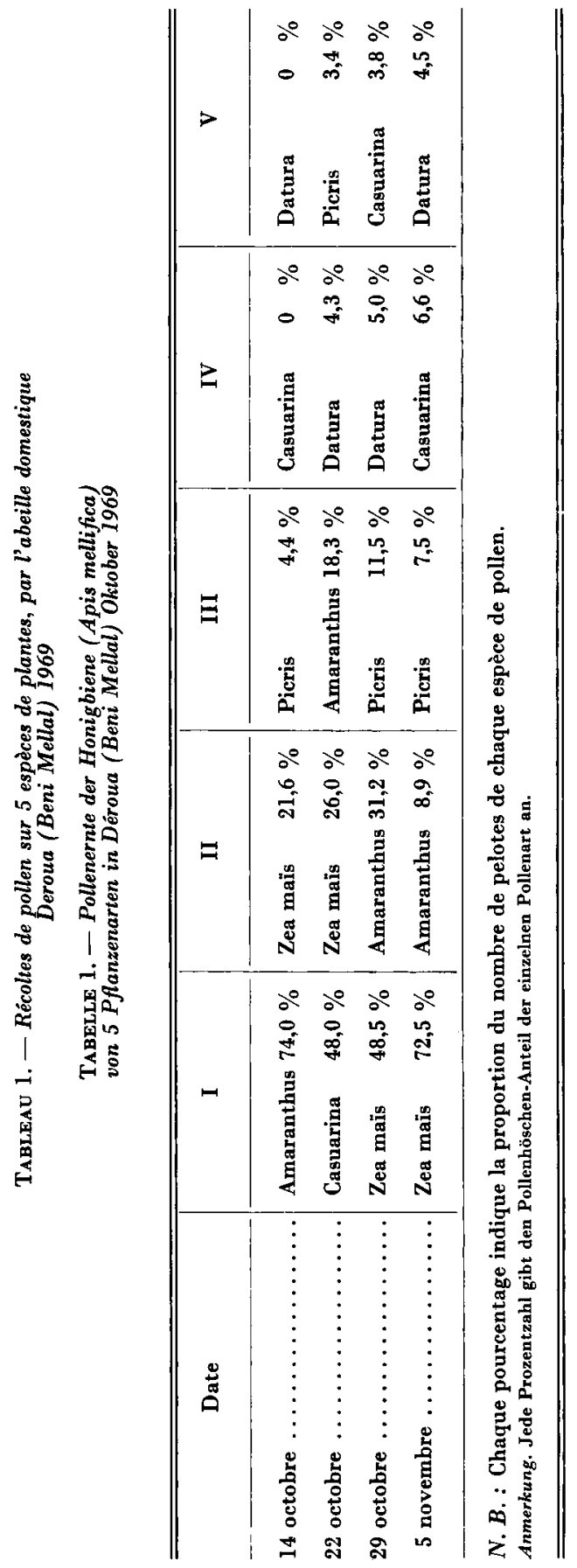


la récolte du pollen s'effectue à des heures différentes pour chaque espèce de plante (fig. 7).

\section{Datura est récolté entre 8.30 et 9.30}

Picris « « « 8.30 et 10.30

Maïs et Amaranthus « 9.30 et 11.30

Casuarina « $\quad$ (c 10.30 et 12.30
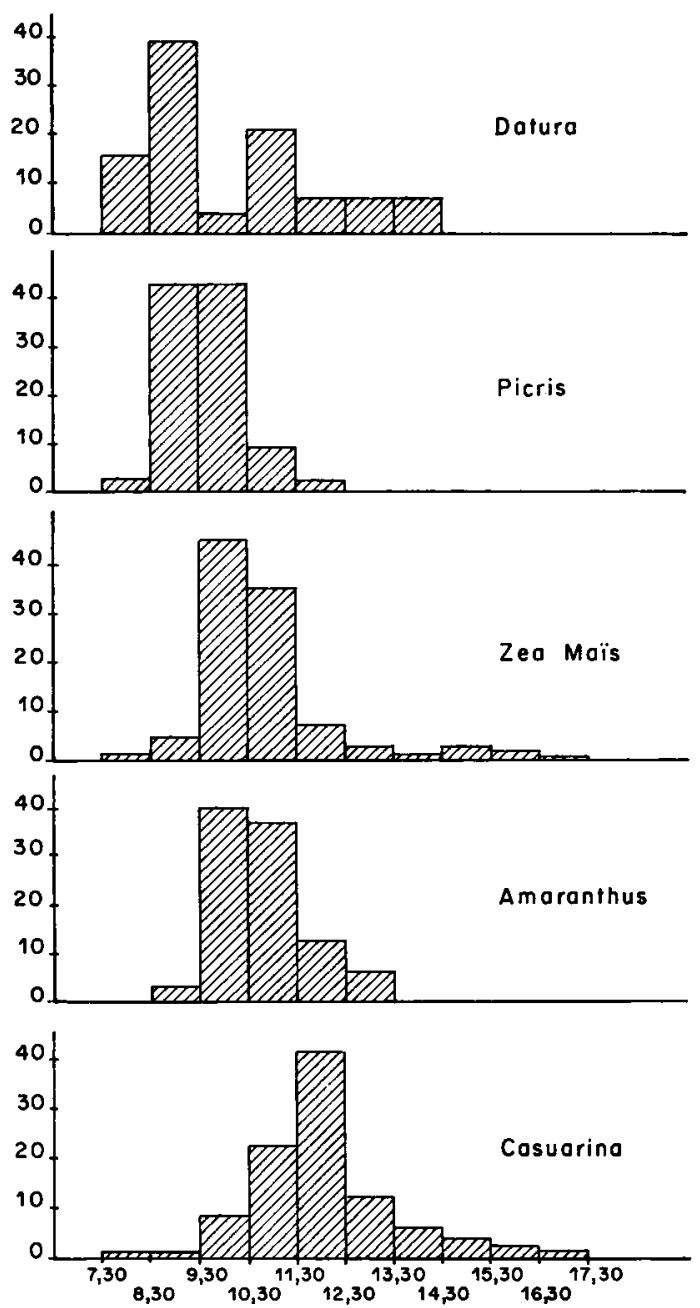

Fig. 7. - Horaire de récolte (axe des $x$ ) des 5 plantes compétitrices principales, par une colonie originaire de Rabat Déroua (Beni Mellal) octobre 1969.

Axe des $y=$ Pourcentage du nombre de pelotes récoltées en 10 heures.

Aвв. 7. - Sammel-Stundenplan ( $x$-Achse) der 5 hauptsächlich in betracht kommenden Pfanzen eines aus Rabat stammenden Volkes, Oktober 1969 :

y-Achse = Durchschnittl. Zahl der in 10 Stdn. gesammelten Pollenhöschen. 


\section{v. - DISCUSSION}

Notre évaluation moyenne de la concentration du nectar de luzerne correspond à celles de Vansell et Todd (1946) et de Petkov (1967). Ces auteurs constatent aussi qu'il existe une corrélation négative entre la concentration et l'humidité de l'air et du sol. Cependant, le rôle joué par la concentration dans l'attractivité du nectar pour les abeilles est jugé négligeable par Pedersen (1953), tandis que Vansell et Todo (1946) estiment que les luzernes peu irriguées ont un nectar concentré attirant un plus grand nombre de butineuses de pollen. Nous ne pouvons pas porter de jugement car la concentration n'a guère varié du premier au dernier jour d'observation. Nous pouvons seulement affirmer que la variation de la concentration au cours de la journée n'influe pas sur la fréquentation des fleurs par les butineuses (fig. 4).

La quantité de nectar est considérée comme un facteur important de l'attractivité des fleurs (Vanseld, 1941 - Pedersen, 1953). Pour le premier de ces auteurs, ce sont des températures de $26-32^{\circ} \mathrm{C}$ qui permettent la sécrétion la plus abondante; pour le second, c'est un bon ensoleillement qui favorise la production du nectar. Il est permis de penser que la diminution du nombre de butineuses pendant le mois d'octobre est due à l'abaissement des températures qui a ralenti la sécrétion de nectar. De même, on peut supposer que la floraison d'été bénéficiant de températures élevées fournit un nectar plus abondant et attire un plus grand nombre de butineuses de pollen.

La durée journalière du butinage est longue et comparable à celle qu'Obrtel et Sedivy (1965) ont observée, soit environ 8 heures avec un maximum d'activité en fin de matinée. Le pourcentage des butineuses de pollen est assez exceptionnel si on le compare aux données de divers auteurs. Comme le remarque Reinhardt (1952) ces butineuses n'ont pas le comportement de déclenchement stéréotypé des abeilles solitaires (Megachile, Eucera, Melitta, Andrena, etc.), qui visitent seulement les fleurs vierges et qui adoptent obligatoirement la position frontale sur les fleurs. Selon le même auteur, il existe certaines abeilles qui récoltent à la fois le pollen et le nectar et ont un pouvoir de déclenchement inférieur à celui des butineuses de pollen. Cette description semble correspondre à notre type de butineuses. La rapidité de butinage de nos abeilles est inférieure à celle des butineuses de nectar évaluée à 10-15 fleurs/mn. (Reinhardt, 1952 - Lecomte, 1959, etc.) et à celle des butineuses de pollen évaluée à 6-7 fleurs/mn (VANsell et Todd, 1946...). Le pourcentage des déclenchements qu'elles provoquent est aussi inférieur à celui des butineuses de pollen et des butineuses de nectar « inexpérimentées » (Moczar, 1961 - BoJTös, 1966) mais supérieur à celui des butineuses de nectar “ expérimentées ". L'efficacité pollinisatrice des abeilles domestiques de la 
région de Beni Mellal est modeste mais non négligeable si l'on tient compte des possibilités d'accroître le nombre de butineuses par un apport de colonies dans les champs de luzerne.

Le comportement de la colonie originaire de la région de Rabat, opposé à celui des ruches locales, peut être expliqué. Les observations de Louveaux et al. (1966) ont mis en évidence l'existence d'écotypes conservant après déplacement dans une région étrangère, leurs caractéristiques de butinage adaptées au régime bioclimatique d'origine. L'existence d'un ( écotype Rabat ) et d'un “ écotype Beni Mellal » n'est qu'hypothétique. Hare et Vansell (1946) recommandent en effet de ne pas se fier aux résultats obtenus à partir d'une seule ruche et d'une seule année. Quant à LovvEaux (1958) il signale le comportement “ individualiste » vis-à-vis des plantes pollinifères, des ruches appartenant à un rucher d'une même race géographique. On constate que certaines ruches ont une affinité marquée pour un pollen ou une famille de plantes et que cette affinité se conserve d'une année à l'autre (Louveaux, 1967). D'autre part, des expériences entreprises aux U.S.A. ont démontré que l'affinité pour le pollen de luzerne est un caractère héréditaire, indépendant du caractère « affinité pour le nectar » et probablement supporté par plusieurs gènes (Nye et Mackensen 1965 - Mackensen et Nye, 1966). Le déplacement de colonies sélectionnées pour la récolte du pollen de luzerne d'une région favorable à une région défavorable à cette récolte atténue mais n'efface pas le caractère “ affinité pour le pollen de luzerne ) (Nye et Mackensen, 1970).

Avec les réserves émises plus haut, notre hypothèse est la suivante : il existe un écotype d'abeille domestique dans la région relativement humide de Rabat qui n'a pas d'affinité pour le pollen de luzerne, d'autres écotypes, dans les régions arides de Settat et de Beni Mellal qui ont une affinité certaine pour ce pollen.

\section{VI. - CONCLUSION}

En 1969, dans les régions de Settat et de Beni Mellal, la luzerne bénéficie de l'action pollinisatrice des abeilles domestiques locales. La population des ouvrières récoltant le pollen représentait :

- en juin, à Settat $9 \%$ des butineuses,

— en octobre, à Beni Mellal, $86 \%$ des butineuses.

A Beni Mellal, les caractéristiques du butinage étaient les suivantes :

- Durée d'activité journalière : 8 heures avec un maximum entre 9 heures 30 et 12 heures 30 .

- Vitesse de butinage : 4 fleurs par minute.

- Pourcentage de déclenchement : $20 \%$ du total des fleurs visitées (30\% des fleurs vierges visitées). 
Dans la région de Rabat, les abeilles domestiques n'ont eu aucune activité pollinisatrice sur la luzerne. On suppose que l'aridité des régions de Beni Mellal et Settat est plus favorable à la pollinisation de la luzerne par l'abeille domestique que le climat plus tempéré de la zone littorale de Rabat. La chaleur rend en effet plus attractives les fleurs de luzerne en accroissant la sécrétion du nectar et la sécheresse des terrains non irrigués ne permet pas le développement de plantes pollinifères compétitrices. Une affinité génétique des écotypes de régions arides pour le pollen de luzerne pourrait aussi jouer un rôle.

Sur ce dernier point, des questions restent en suspens :

- Le comportement de la ruche de Rabat transportée à Beni Mellal est-il représentatif de celui de " l'écotype Rabat "?

- Ge comportement est-il le même en juillet et en octobre et se conservet-il d'une année à l'autre?

- Les écotypes des régions arides conservent-ils leur comportement après déplacement dans une région littorale?

L'efficacité pollinisatrice de l'abeille domestique sur la luzerne dans certaines régions du Maroc devrait susciter des recherches nouvelles, afin d'élucider le problème des races géographiques marocaines et de justifier ou non l'emploi de ruches originaires d'une région bioclimatique différente de celle où se trouve la luzerne à polliniser.

Reçu pour publication en juin 1971.

Eingegangen im Juni 1971.

\section{REMERCIEMENTS}

Je tiens à remercier M. MATHEz (I.S.C. Rabat) qui a déterminé les plantes récoltées sur le terrain d'observation. Je remercie M. LouveauX (I.N.R.A., Bures-sur-Yvette) et Mme VAN CAmpo (Laboratoire de Palynologie de Montpellier, C.N.R.S.) pour l'aide reçue lors de l'analyse des préparations polliniques.

\section{ZUSAMMENFASSUNG}

Die fehlende Affinität der Honigbiene (Apis mellifica L.) zum Luzernepollen wird allgemein mit dem beim “ Aufklinken 》 der Blüten verursachten Rückstoss der Staubfäden und mit dem Fehlen attraktiver Substanzen im Pollen der Luzerne erklärt. Unter gewissen landwirtschaftlichen Bedingungen können die Sammelbienen bei der Samenbildung der Luzerne eine Rolle spielen (BoHART 1957, Lecomte 1959).

Wir konnten einige Erfahrungen über das Bestäubungsverhalten von Apis mellifica intermissa in den marokkanischen Luzernefeldern sowie über gewisse, die Sammeltätigkeit beeinflussende Faktoren sammeln. 
In der gemässigteren Küstenregion von Rabat wurden die Honigbienen niemals beim “ Aufklinken » von Luzerneblüten und auch nicht beim Bilden von Pollenhöschen aus Luzernepollen beobachtet. Im Dürregebiet von Settat, das weniger reich an Pollenspendern ist, hatten nahezu $10 \%$ der die Luzerne be fliegenden Bienen Höschen aus Luzernepollen.

\section{1. - Material und Methoden}

Eingehende Beobachtungen wurden auf dem Versuchsgut von Déroua in der Nähe von Beni Mellal (Tadla) bei später Blütezeit der Luzerne im Oktober durchgeführt. Die Bienen wurden an fünf Tagen, am 7., 14., 22., 29. Oktober und am 5. November 1969 auf einer 11 ha grossen bewässerten Fläche beobachtet (Südafrikanische Art). Die dort vorhandenen Bienen stammten aus einem $300 \mathrm{~m}$ vom Feld entfernten Bienenstand. Am 7. Oktober stellten wir eine Dadantbeute mit Pollenfalle aus einem Bienenstand von Rabat am Rande des Feldes auf.

Ein Teil der Beobachtungen bezog sich auf die Luzerneblüten : Entwicklung der Dichte der Blütenstände sowie der Konzentration des Nektars in der Trockenmasse. Ferner wurde stündlich die Anzahl der Sammelbienen auf einem blühendem Streifen von $100 \times 1 \mathrm{~m}$ gezählt (s. Lecomte 1962 und Beneder 1970). Weiter berechneten wir den Anteil der Bienen mit Höschen aus Luzernepollen und analysierten das Sammelverhalten von 50 Arbeiterinnen. Schliesslich wurde stündlich der Inhalt der Pollenfalle entnommen, Höschen für Höschen ausgelesen und analysiert. Die Pollenbestimmung wurde nach der Methode von Maurizio und Louveaux (1965) ausgeführt. Während der Beobachtungszeit wurden Temperatur und Luftfeuchtigkeit in der Wetterhütte registriert.

\section{2. - Ergebnisse}

— Die Dichte der Blütenstände blieb sehr gering (19 auf $25 \mathrm{~m}^{2}$ ).

- Die von den Blüten abgesonderte Nektarmenge schien mit abnehmenden Nachttemperaturen geringer zu werden (Abb. 2). Die Durchschnittskonzentration des Nektars hat sich kaum verändert $(35-43 \%)$. Im Tagesablauf verändert sich die Konzentration umgekehrt proportional zur Luftfeuchtigkeit (Abb. 3).

- Die Zahl der Sammelbienen verringerte sich vom 14. Oktober ab, der Anteil der Pollensammlerinnen blieb jedoch stabil (81-94\%).

- Die Sammelgeschwindigkeit betrug 4 Blüten je Minute. $20 \%$ der besuchten Blüten wurden " aufgeklinkt » (Abb. 5).

— Die Pollenfalle ergab eine ziemlich magere Ausbeute (Maximum $=606$ Pollenhöschen am Tag) und enthielt kein einziges Luzernepollenhöschen. Am Bienenstock wurden folgende Pollenarten gesammelt :

Leitpollen : Zea maïs, Casuarina, Amaranthus, Picris und Datura.

Begleitpollen : Convolvulus, Hibiscus, Gossypium, Lactuca, Erigeron, Eucalyptus, Solanum, Hirschfeldia, Bougainvillea (Abb. 6 u. 7).

Diese Pflanzen wurden auf dem Gut gezogen, oder sie wuchsen auf den bewässerten Parzellen.

\section{3. - Schlussfolgerungen}

Es muss eine Korrelation zwischen der Abnahme der Sammeltätigkeit der Honigbiene auf der Luzerne im Verlauf der Beobachtungszeit und dem mit dem nächtlichen Temperaturrückgang verbundenen Absinken der Nektarsekretion bestehen. Der Anteil der Pollensammlerinnen war ausnehmend hoch, die Sammelgeschwindigkeit und der Prozentsatz des (Aufklinkens » jedoch ziemlich gering (s. Reinhakd 1952, Lecomte 1959, VANSELL und TodD 1946, Moczar 1961 usw...). Wir vermuten, dass die Trockenheit der Gebiete von Settat und 
Beni Mellal die Bestäubung der Luzerne durch die Honigbiene mehr als das gemässigtere Klima von Rabat begünstigt. Verschiedene Faktoren können im Dürregebiet eine Rolle spielen : die von der Luzerne abgesonderte Nektarmenge ist grösser, die Pollenquellen sind weniger zahlreich, eine genetische Affinität der Ökotypen der Biene für den Luzernepollen in den Trockengebieten kann ebenfalls von Bedeutung sein (Louveaux 1967, Nye u. Mackensen 1970). Das zuletzt Angeführte bedarf noch des Beweises, denn es wäre leichtfertig, aufgrund eines einzigen Beobachtungsjahres und des Verhaltens eines einzelnen Volkes eine Schlussfolgerung zu ziehen.

\section{REFÉRENCES BIBLIOGRAPHIQUES}

Anasiewicz A. et Warakomska Z., 1969. Occurence of Bumble bees on alfalfa (M. media) in the province of Lublin and pollen analysis of their pollen loads. Ekol. Pol. ser A., t. 17, 587-609.

BENEDEK P., 1970. Effects of plot size, stand density and state of flowering on wild bees pollinating lucerne. Acta Agron. Acad. Sc. Hung., t. 19 (3-4), 283-292.

Bohant G.-E., 1957. Pollination of alfalfa and red clover. Ann. Rev. Entomol., t. 2, $255-380$.

Bostös Z., 1966. Honey bee activity in alfalfa (M. Sativa L.) pollination in Hungary. Acta Agron. Acad. Sc. Hung, t. 15 (1-2), 215-221.

Hare Q.-A. et Vansell G.-H., 1946. Pollen collection by honey bees in the Delta, Utah, alfalfa seed producing area. J. Amer. Soc. Agron., 38, 462-469.

Lecomтe J., 1959. Luzerne et Apiculture. Ann. Abeille, 2 (3), 211-221.

Lecomte J., 1959. Premières observations sur le comportement des insectes pollinisateurs de la luzerne. Ann. Abeille, 2 (4), 277-284.

Lecomte J., 1962. Techniques d'étude des populations d'insectes pollinisateurs; Ann. Abeille 5 (3), 201-213.

Louveaux J., 1958. Recherches sur la récolte du pollen par les abeilles (Apis mellifica L.). Ann. Abeille, 1 (3), 113-188 et 1 (4), 197-221.

Louveaux J., 1959. (même titre) Ann. Abeille, 2 (1), 13-111.

Louveaux J. et al, 1966. Les modalités de l'adaptation des abeilles (Apis mellifica L.) au milieu naturel. Ann. Abeille, 9 (4), 323-350.

Louveaux J., 1967. Les problèmes posés par la génétique et la sélection de l'abeille. Ann. Abeille, 10 (4), 213-252.

Mackensen O. et NyE W.-P., 1966. Selecting and breeding honey bees for collecting alfalfa pollen. J. of Apicult. Research 5 (2), 79-86.

Maurizio A. et Louveaux J., 1965. Pollens des plantes mellifères d'Europe. U.G.A.F., 38, bd Sebastopol Paris IVe.

Moczar L., 1961. The role of the honeybee (Hym. Apis mellifica L.) in relation to lucerne in Hungary. Acta entomolgica Muséi Nationalis Pragae, XXxiv, 571, 5-11.

NyE W.-P. et Mackensen O., 1965. Preliminary report selection and breeding of honeybees for alfalfa pollen collection. J. of apic. Res. 4 (1), 43-48.

NyE W.-P. et Mackensen O., 1970. Selective breeding of honey bees for alfalfa pollen collection with tests in high and low alfalfa pollen collection regions. J. of Apic. Res., 9 (2), $61-64$.

Obrtel R. et Sedivy J., 1965. Insects pollinating lucerne (M. sativa) in Czeckoslovakia. Zool. Listy, 14 (4), 338-348.

Pedensen M.-W., 1953. Environmental factors affecting nectar secretion and seed production in alfalfa. Agron. $J ., 8,359-361$.

Pexkov V. et Srminchiev T., 1967. Study on the role of bees in lucerne pollination. Rasteniev Nauki, 4 (5), 81-92. 
RinnundT J.F., 1952. Some reponses of honey bees to alfalfa flowers. Amer. Nat. 86 (830), 257-275.

Srzuckandt R., 1962. Untersuchungen über die Wirksamkeit von Honigbienen Apis mellifica bei der Luzerne bestäubung. $Z$. Pflanzensüchtung, 47, 15-50.

TA8si J.-N., sous-presse. In fluence de la date de la précoupe de la luzerne (M. sativa L.) sur sa pollinisation. Apidologie.

VANskLL G.-H., 1941. Alfalfa nectar and the honey bees. J. Econ. Eniomol. 34, 21-23.

Varsedx G.-H. et Todd F.-E., 1946. Alfalfa tripping by insects. J. Amer. Soc. Agr., 38, 470-488. 